International Journal of Algebra, Vol. 1, 2007, no. 12, 587 - 600

\title{
The Calculation of Represenations of the Group of Overhand Knot
}

\author{
Ahmet Küçük \\ Atatürk Üniversitesi, Fen-Edebiyat Fakültesi \\ Matematik Bölümü, Erzurum, Turkey \\ akucuk@atauni.edu.tr
}

\begin{abstract}
This paper is a continuation of earlier Works of the author $[1,2]$. The Group of overhand knot is given [2] by

$$
G=|x, y: x y x=y x y|
$$

The symmetric group of degree four is denoted by $S_{4}$ and is given as follows [4] :

$$
S_{4}=\left|a, b: a^{3}=b^{4}=(a b)^{2}=1\right|
$$

where $a=(234)$ and $b=(243)(12)$ Here, the complete classification of $G$ in $S_{4}$ is given. These calculations are mostly obtained in [6]. These representations are divided into eight non-equivalent classes. For each class a representative homomorphism is considered and the corresponding fundamental group of 4-sheeted covering space of the overhand knot has been calculated by the algorithm of Professor Fox.
\end{abstract}

\section{Mathematics Subject Classification: 22A30}

Keywords: Overhand, Knot

\section{Preliminaries}

A planer normal projection of the overhand knot $\mathrm{K}$ is given [6]. The fundamental group of the complement of $\mathrm{K}$ in 3-sphere, i.e. $G=\pi\left(S^{3}-K\right)$, is called the group of overhand knot. By means of Wirtinger's method $G$ can be calculated as 


$$
G=\left|x, y: x^{3}=y^{4}=(x y)^{2}=1\right|
$$

As it is well known the group $G$ is an infnite group of deficiency 1 . The symmetric group of order 24 is given as [4]

$$
S_{4}=\left|x, y: x^{3}=y^{4}=(x y)^{2}=1\right|
$$

where $x=(234)$ and $y=(243)(12)$. To find representations of $G$ in $S_{4}$ depends on the following theorem $[9, \mathrm{p} .11]$

Theorem. If $|a, b, c, \ldots: P, Q, R, \ldots|$ is apresentation of a group $G$ and if $x, y, z, \ldots$ are elements of a group $\mathrm{H}$ such that $P(x, y, z, \ldots), Q(x, y, z, \ldots)$,

$R(x, y, z, \ldots), \ldots$ is each the identity element in $\mathrm{H}$, then the group $\mathrm{G}$ can be mapped homomorphically into $\mathrm{H}$ under the obvious homomorphism.

Two homomorphisms $f, g: C \rightarrow S_{4}$ are called equivalent if there exists a permutation $p \in S_{4}$ such that for all $w \in G, f(w)=p \cdot g(w) \cdot p^{-1}$.

\section{Representation of $G$ in $S_{4}, h: G \rightarrow S_{4}$}

The group of overhand knot is given [2] by

$$
G=|x, y: x y x=y x y|
$$

The symmetric group of degree four is denoted by $S_{4}$ and is given as follows [4] :

$$
S_{4}=\left|a, b: a^{3}=b^{4}=(a b)^{2}=1\right|
$$

where $a=(234)$ and $b=(243)(12)$. Here, the complete classification of $G$ in $S_{4}$ is given. These calculations are mostly obtained in [6]. These representations are divided into eight non-equivalent classes. For each class a representative homomorphism is considered and the corresponding fundamental group of 4-sheeted covering space of the overhand knot has been calculated by the algorithm of Professor Fox.

Class 1: Cyclic homomorphisms[6]

$$
h(x)=h(y)=(1234)
$$




$$
\begin{aligned}
& h(x)=h(y)=(1243) \\
& h(x)=h(y)=(1324) \\
& h(x)=h(y)=(1342) \\
& h(x)=h(y)=(1423) \\
& h(x)=h(y)=(1432)
\end{aligned}
$$

Class 2: Almost cyclic homomorphisms[6]

$$
\begin{aligned}
& h(x)=(1234), h(y)=(1243) \\
& h(x)=(1243), h(y)=(1234) \\
& h(x)=(1243), h(y)=(1432) \\
& h(x)=(1432), h(y)=(1243) \\
& h(x)=(1234), h(y)=(1342) \\
& h(x)=(1342), h(y)=(1234)
\end{aligned}
$$




$$
\begin{aligned}
& h(x)=(1234), h(y)=(1423) \\
& h(x)=(1423), h(y)=(1234) \\
& h(x)=(1234), h(y)=(1324) \\
& h(x)=(1324), h(y)=(1234) \\
& h(x)=(1243), h(y)=(1324) \\
& h(x)=(1432), h(y)=(1342) \\
& h(x)=(1324), h(y)=(1243) \\
& h(x)=(1423), h(y)=(1243) \\
& h(x)=(1243), h(y)=(1423)
\end{aligned}
$$




$$
\begin{aligned}
& h(x)=(1342), h(y)=(1423) \\
& h(x)=(1423), h(y)=(1342) \\
& h(x)=(1324), h(y)=(1432) \\
& h(x)=(1432), h(y)=(1324) \\
& h(x)=(1432), h(y)=(1423) \\
& h(x)=(1423), h(y)=(1432)
\end{aligned}
$$

Class 3 : Interesting homomorphisms [6]

$$
\begin{aligned}
& h(x)=(123)(4), h(y)=(142)(3) \\
& h(x)=(123)(4), h(y)=(134)(2) \\
& h(x)=(123)(4), h(y)=(243)(1) \\
& h(x)=(132)(4), h(y)=(124)(3) \\
& h(x)=(132)(4), h(y)=(124)(3)
\end{aligned}
$$




$$
\begin{aligned}
& h(x)=(132)(4), h(y)=(143)(2) \\
& h(x)=(124)(3), h(y)=(234)(1) \\
& h(x)=(124)(3), h(y)=(143)(2) \\
& h(x)=(124)(3), h(y)=(132)(4) \\
& h(x)=(142)(3), h(y)=(243)(1) \\
& h(x)=(143)(2), h(y)=(243)(1) \\
& h(x)=(142)(3), h(y)=(134)(2) \\
& h(x)=(134)(2), h(y)=(243)(1) \\
& h(x)=(142)(3), h(y)=(132)(4)
\end{aligned}
$$




$$
\begin{aligned}
& h(x)=(143)(2), h(y)=(132)(4) \\
& h(x)=(234)(1), h(y)=(143)(2) \\
& h(x)=(234)(1), h(y)=(124)(3) \\
& h(x)=(234)(1), h(y)=(132)(4) \\
& h(x)=(243)(1), h(y)=(134)(2) \\
& h(x)=(243)(1), h(y)=(142)(3) \\
& h(x)=(243)(1), h(y)=(123)(4)
\end{aligned}
$$

Note : $h(x)=(1)(234), h(y)=(2)(134)$ is not a representation of $G$ in $S_{4}$. For, $(x y x \bar{y} \bar{x} \bar{y})$ can not be map onto identity permutation under $h[6]$.

Class 4 : The trivial homomorphism[6]

$$
\begin{gathered}
h(x)=h(y)=(1)(2)(3)(4) \\
\text { Class } 5[6] \\
h(x)=h(y)=(12)(34) \\
h(x)=h(y)=(13)(24) \\
h(x)=h(y)=(14)(23)
\end{gathered}
$$


Class 6: Three cyclic homomorphisms [6]

$$
\begin{aligned}
& h(x)=h(y)=(123)(4) \\
& h(x)=h(y)=(132)(4) \\
& h(x)=h(y)=(124)(3) \\
& h(x)=h(y)=(142)(3) \\
& h(x)=h(y)=(134)(2) \\
& h(x)=h(y)=(143)(2) \\
& h(x)=h(y)=(243)(1) \\
& h(x)=h(y)=(234)(1)
\end{aligned}
$$

Class 7 : Two cyclic homomorphisms[6]

$$
\begin{aligned}
& h(x)=h(y)=(12) \\
& h(x)=h(y)=(13) \\
& h(x)=h(y)=(14)
\end{aligned}
$$




$$
\begin{aligned}
& h(x)=h(y)=(23) \\
& h(x)=h(y)=(24) \\
& h(x)=h(y)=(34)
\end{aligned}
$$

Class 8: Irregular homomorphisms. The corresponding branched covering space of overhand knot is always the three sphere [6].

$$
\begin{aligned}
& h(x)=(12)(3), h(y)=(13)(2) \\
& h(x)=(13)(2), h(y)=(12)(3) \\
& h(x)=(13)(2), h(y)=(23)(1) \\
& h(x)=(23)(1), h(y)=(13)(2) \\
& h(x)=(23)(1), h(y)=(12)(3) \\
& h(x)=(12)(3), h(y)=(23)(1) \\
& h(x)=(12)(4), h(y)=(14)(2) \\
& h(x)=(14)(2), h(y)=(12)(4)
\end{aligned}
$$




$$
\begin{aligned}
& h(x)=(14)(2), h(y)=(24)(1) \\
& h(x)=(24)(1), h(y)=(14)(2) \\
& h(x)=(24)(1), h(y)=(12)(4) \\
& h(x)=(12)(4), h(y)=(24)(1) \\
& h(x)=(13)(4), h(y)=(34)(1) \\
& h(x)=(24)(3), h(y)=(23)(4) \\
& h(x)=(34)(1), h(y)=(13)(4) \\
& h(x)=(14)(3), h(y)=(13)(4) \\
& h(x)=(13)(4), h(y)=(14)(3)
\end{aligned}
$$




$$
\begin{aligned}
& h(x)=(34)(2), h(y)=(24)(3) \\
& h(x)=(24)(3), h(y)=(34)(2) \\
& h(x)=(34)(2), h(y)=(23)(4) \\
& h(x)=(23)(4), h(y)=(34)(2)
\end{aligned}
$$

\section{Examples}

In the above classification only the examples of classes $1,2,3$ are considared here. The others are related to disconnected coveaings and some of them are already studied in [6].

a. Cyclic four sheeted covering space of Overhand Knot

$$
\begin{aligned}
& h: G \rightarrow S_{4}, h(x)=h(y)=(1234), \\
& x y x, \bar{y} \bar{x} \bar{y} \\
& 1234321 \\
& 2341432 \\
& 3412143 \\
& 4123214
\end{aligned}
$$

Let $\sum$ be the branced covering related to this homomorphism. If $L$ is the branch curve over $K$ in $\sum$, then $\sum-L$ is a four sheeted cyclic covering space of $S^{3}-K$. By Fox algorithm and from the above table the free product group $\pi\left(\sum-K\right) * F_{3}$ is calculated as follows

$$
\pi\left(\sum-K\right) * F_{3}=\left|\begin{array}{l}
x_{1}, y_{1}: x_{1} y_{2} x_{3} \bar{y}_{3} \bar{x}_{2} \bar{y}_{1} \\
x_{2}, y_{2}: x_{2} y_{3} x_{4} \bar{y}_{4} \bar{x}_{3} \bar{y}_{2} \\
x_{3}, y_{3}: x_{3} y_{4} x_{1} \bar{y}_{1} \bar{x}_{4} \bar{y}_{3} \\
x_{4}, y_{4}: x_{4} y_{1} x_{2} \bar{y}_{2} \bar{x}_{1} \bar{y}_{4}
\end{array}\right|
$$

where $F_{3}$ is a free group of rank $3 \cdot[1,6,7,8]$. 
As generators of $F_{3}$ we choose $y_{2}, y_{3}, y_{4}$ and adjoining $y_{2}=y_{3}=y_{4}=1$ to the above group we get

$$
\pi\left(\sum-K\right)=\left|\begin{array}{l}
x_{1}, x_{1}, x_{3}: x_{1} x_{3} \bar{x}_{2} \bar{y}_{1}, x_{2} x_{4} \bar{x}_{3} \\
x_{4}, y_{1}: x_{3} x_{1} \bar{y}_{1} \bar{x}_{4}, x_{4} y_{1} x_{2} \bar{x}_{1}
\end{array}\right|
$$

The branch relation is $y_{1} y_{2} y_{3} y_{4}=1\left(\right.$ or $\left.x_{1} x_{2} x_{3} x_{4}=1\right)$ which reduces to $y_{1}=1$. Upon adjoining $y_{1}=1$ to $\pi\left(\sum-K\right)$ we get

$$
\begin{array}{r}
\pi\left(\sum\right)=\left|x_{i}: x_{i+2} \bar{x}_{i+1}, i=1,2,3,4(\bmod 4)\right| . \\
x_{4}=x_{3} x_{1}, x_{2}=x_{1} x_{3}, x_{1}=a \text { and } x_{3}=b \text { are used to get } \\
\pi\left(\sum\right)=\left|a, b: a b^{2} a \bar{b}, b a^{2} b \bar{a}\right|
\end{array}
$$

Then, we obtain $H_{1}\left(\sum\right)=\left|a, b: b=a^{-2}, a=b^{-2}\right|$.or $H_{1}\left(\sum\right)=\left|a: a^{3}\right|$.

b. Cyclic four sheeted almost cylic covering of Overhand Knot $[6,7,8]$

$$
\begin{aligned}
& h: G \rightarrow S_{4}, h(x)=(1234), h(y)=(1243) . \\
& \text { x y } x, \bar{y} \bar{x} \bar{y} \\
& 1241321 \\
& 2312142 \\
& 3434213 \\
& 4123434
\end{aligned}
$$

Let $\Omega$ be the branched covering space related to this homomorphism. Let $M$ be the branch curve over Overhand knot. By calculations similar to those above we obtain

$$
\Omega\left(\sum-M\right) * F_{3}=\left|\begin{array}{l}
x_{1}, y_{1}: x_{1} y_{2} x_{4} \bar{y}_{3} \bar{x}_{2} \bar{y}_{1} \\
x_{2}, y_{2}: x_{2} y_{3} x_{1} \bar{y}_{1} \bar{x}_{4} \bar{y}_{2} \\
x_{3}, y_{3}: x_{3} y_{4} x_{3} \bar{y}_{2} \bar{x}_{1} \bar{y}_{3} \\
x_{4}, y_{4}: x_{4} y_{1} x_{2} \bar{y}_{4} \bar{x}_{3} \bar{y}_{4}
\end{array}\right|
$$

Choosing $x_{1}, x_{3}, x_{4}$ as generators of $F_{3}$ and adding $x_{1}=x_{3}=x_{4}=1$ to the above group we obtain 


$$
\Omega\left(\sum-M\right)=\left|\begin{array}{l}
x_{1}, y_{1}, y_{2}: x_{1} y_{2} \bar{y}_{3} \bar{y}_{1}, y_{3} x_{1} \bar{y}_{1} \bar{y}_{2} \\
y_{3}, y_{4}: y_{4} \bar{y}_{2} \bar{x}_{1} \bar{y}_{3}, y_{1} \bar{y}_{4}^{2}
\end{array}\right|
$$

The branch relation is $x_{1} x_{2} x_{3} x_{4}=1$,i.e. $x_{1}=1\left(\right.$ or $\left.y_{1} y_{2} y_{3} y_{4}=1\right)$. When we add $x_{1}=1$ to $\pi(\Omega-M)$, we get [9]

$$
\pi(\Omega)=\left|y_{1}, y_{2}, y_{3}, y_{4}: y_{2} \bar{y}_{3} \bar{y}_{1}, y_{3} \bar{y}_{1} \bar{y}_{2}, y_{4} \bar{y}_{2} \bar{y}_{3}, y_{1} \bar{y}_{4}^{2}\right|
$$

Using $y_{2}=\bar{y}_{3} y_{4}$ and $y_{1}=y_{4}^{2}$

$$
\pi(\Omega)=\left|y_{3}, y_{4}: \bar{y}_{3} y_{4} \bar{y}_{3} \bar{y}_{4}^{2}, y_{3} \bar{y}_{4}^{2} \bar{y}_{4} y_{3}\right|
$$

or putting $y_{4}=a$ and $y_{4}=b$ we obtain

$$
\pi(\Omega)=\left|a, b: \bar{a} b \bar{a}=b^{2}, a^{2}=b^{3}\right| .
$$

From this we get [9]

$$
H_{1}(\Omega)=\left|a, b: b=a^{-2}, a^{2}=b^{3}\right|=\left|a: a^{8}\right|=Z_{8} .
$$

c. An interesting four sheeted covering of overhand knot $[6,7,8,9,2,3]$

$$
\begin{aligned}
& h: G \rightarrow S_{4}, h(x)=(123)(4), h(y)=(142)(3) . \\
& x y x, \bar{y} \bar{x} \bar{y} \\
& 1212441 \\
& 2331212 \\
& 3144133 \\
& 4423324
\end{aligned}
$$

Let $\Psi$ and $N$ be the related branched covering and the branch curve over $K$ respectively. Similar calculations lead to the following results:

$$
\Pi(\Psi-N) * F_{3}=\left|\begin{array}{l}
x_{1}, y_{1}: x_{1} y_{2} x_{1} \bar{y}_{4} \bar{x}_{4} \bar{y}_{1} \\
x_{2}, y_{2}: x_{2} y_{3} x_{3} \bar{y}_{2} \bar{x}_{1} \bar{y}_{2} \\
x_{3}, y_{3}: x_{3} y_{1} x_{4} \bar{y}_{1} \bar{x}_{3} \bar{y}_{3} \\
x_{4}, y_{4}: x_{4} y_{4} x_{2} \bar{y}_{3} \bar{x}_{2} \bar{y}_{4}
\end{array}\right|
$$




$$
\begin{gathered}
\Pi(\Psi-N)=\left|\begin{array}{l}
x_{1}, y_{4}, y_{1}: x_{1} y_{2} x_{1} \bar{x}_{4} \bar{y}_{1}, y_{3} \bar{y}_{2} \bar{x}_{1} \bar{y}_{2} \\
y_{2}, y_{3}: y_{1} x_{4} \bar{y}_{1} \bar{y}_{3}, x_{4} \bar{y}_{3}
\end{array}\right|, \\
\pi(\Psi)=\left|x, y:(x y)^{3}(y x)^{-3}\right| .
\end{gathered}
$$

Hence $H_{1}(\Psi)=0 .[9]$

\section{References}

[1] BOZHÜYÜK, M.E. On 3-sheeted covering spaces of (3,5)-Turk's Head Knot, Topology and Applications, Colloq. Math. Soc. J. Bolyai 41, Nort Holland (Amsterdam, 1985), 119-124.

[2] BOZHÜYÜK, M.E. Dallanmış Örtü Uzayları Teorisi ve Üç Boyutlu Kürenin Bazı Türk Düğümleri Üzerinde Dallanmış Örtü Uzayları, Profesörlük tezi, Atatürk Üniversitesi(Erzurum, 1983).

[3] BOZHÜYÜK, M.E. On covering spaces of trefoil knot, J. Fac. Sci. KTÜ 2 , (1979), 31-40.

[4] COXTER, H.S.M. and MOSER, W.O.J. Generators and relations for discrete groups, Springer (Berlin, 1972).

[5] CROWELL, R.H. and FOX, R.H. Introduction to Knot Theory, BlaisdellGinn (New York, 1963).

[6] KÜÇÜK, A. (2,2) ve (3,3)-Türk Saadet Dügüumlerinin Örtü Uzayları, Doktora tezi, Atatürk Üniversitesi(Erzurum, 1989).

[7] KÜÇÜK, A. The Fundamental Group of Bordered Surfaces, Bulletin of Pure and Applied Sciences, India, Vol.16E (no2), 101-106, 1997.

[8] KÜÇÜK, A. and BAYRAM, M. Automatic Calculation of the Fundamental group of an oriented surface of genus $n$ with $\mathrm{k}$ boundary surfaces,Applied Mathematics and Computation (2004).

[9] MAGNUS, W., KARRASS, A. and SOLITER, D. Combinatorial Group Theory,Presentation of groups in terms of generators and relations, Interscience (New York, 1966).

Received: June 27, 2007 